\title{
Research on application of industrial intelligent control
}

\author{
Libo Yang \\ Guangdong University of Science \& Technology, Dongguan, Guangdong, 523083, China
}

Keywords: artificial intelligence, industry, intelligent control

\begin{abstract}
In the global tide of artificial intelligence, China has its own specific opportunities. Since the financial crisis, with the market environment, technological progress, factor conditions, the accumulation of capital and other major changes in the situation, China's industrial sector has emerged the phenomenon of differentiation. The fact is that many enterprises have to transform, but also it has brought opportunities to China's industrial production. For example, China's industry can be combined with industrial innovation driven strategy to promote the transformation of the industry. In this article, I will first introduce the background of intelligent control. Then, I will introduce some of the advantages of intelligent control in industry. Furthermore, I would like to analyze the theory in the case of our industrial production. Finally, I put forward the prospect of the development of industrial intelligent control.
\end{abstract}

\section{Introduction}

Intelligent control is the advanced stage of the development of automatic control, artificial intelligence, control theory, information theory, system theory, bionics, evolutionary computation and computer integrated and highly integrated disciplines.It is also an emerging interdisciplinary science. As we all know, intelligent control is a very active and challenging field in the field of automation at home and abroad. It includes not only the automatic control, artificial intelligence, system theory and computer science, but also draw nutrition from biology, is becoming a branch of the field of automation and the rapid development of the most prosperous.

\section{The background of intelligent control}

From the perspective of the development of control theory, the development of this discipline has experienced three main stages, which are the following three stages:

The first stage is from twentieth Century to 40, which is based on the theory of feedback control, which is based on feedback theory, and is a kind of linear control theory. Frequency analysis method based on transfer function, frequency characteristic and root locus. The analysis and design of single input single output, linear fixed length system.

The second stage is from twentieth Century to 60 in the period of "modern control theory", the modern control theory mainly studies the optimal control problem of multivariable system with high performance and high precision. The method includes state space method, Bellman dynamic programming method, Kalman filtering theory and Pontryagin maximum principle. Modern control theory can solve the problem of multi input and multi output, the system can be linear fixed length, can also be nonlinear time-varying.

The third stage is the "big system theory" and "intelligent control theory" period since 1970s. Because of the modern control theory too much reliance on the object mathematical model, the control algorithm design method is very ideal, digital, so in the face of difficult to describe with mathematical models or complicated systems with time-varying, nonlinear and uncertain characteristics, modern control system is incapable of action. In order to improve the quality of the control system and the ability of searching, researchers in the field of control began to consider the application of artificial intelligence technology in the control system. In recent years, researchers in the field of control to the traditional control theory and fuzzy logic, neural network, genetic algorithm and other intelligent technology to make full use of people's experience and knowledge to control the complex system, and gradually formed a new discipline of intelligent control. 


\section{Advantages of intelligent control}

The traditional control method is based on the accurate mathematical model of the controlled object, and the intelligent control is based on the complexity, nonlinearity and uncertainty of the system. The IEEE Control System Association concludes that intelligent control systems must have the ability to simulate human learning and adaptation. Therefore, the use of intelligent control technology in our industrial production process is a very potential thing. An intelligent control system generally should have the following advantages:

\section{Expert control, high degree of specialization}

Expert control developed in the field of artificial intelligence is a kind of intelligent computer program based on knowledge. The essence of expert control is based on the knowledge of the control object and the control law, and the knowledge should be utilized in an intelligent way in order to get the optimized and practical control system. The expert system consists of knowledge base, inference engine, explanation mechanism and knowledge acquisition system. The knowledge base is used to store experiential knowledge, principle knowledge, feasible operation and rules. The knowledge can be modified and expanded by the knowledge acquisition system. According to the system information and the knowledge in the knowledge base, the inference engine is used to solve the current problems. The interpretation mechanism is used to explain the knowledge and provide a user interface. The characteristics of expert control are as follows: professional knowledge in the field of experts, symbolic processing and heuristic reasoning, knowledge acquisition, flexibility, transparency and interactivity.

\section{Neural network control, strong ability to restore}

Neural network control is the use of this tool in the neural network control system, modeling of the complex nonlinear system, it is difficult to describe by conventional methods, or act as a controller, or information processing, and pattern recognition, or fault diagnosis, or several functional groups, the control method of neural network control system is neural network control. Neural network adopts the viewpoint of bionics research on the control of advanced information processing problems in intelligent systems, characteristics of neural network control: it can fully approximate arbitrary nonlinear characteristics; distributed parallel processing mechanism; self-learning and adaptive ability; data fusion; suitable for multi variable system, can be multi variable.

\section{The application of intelligent control technology in the workshop anti-theft system}

\section{System hardware design}

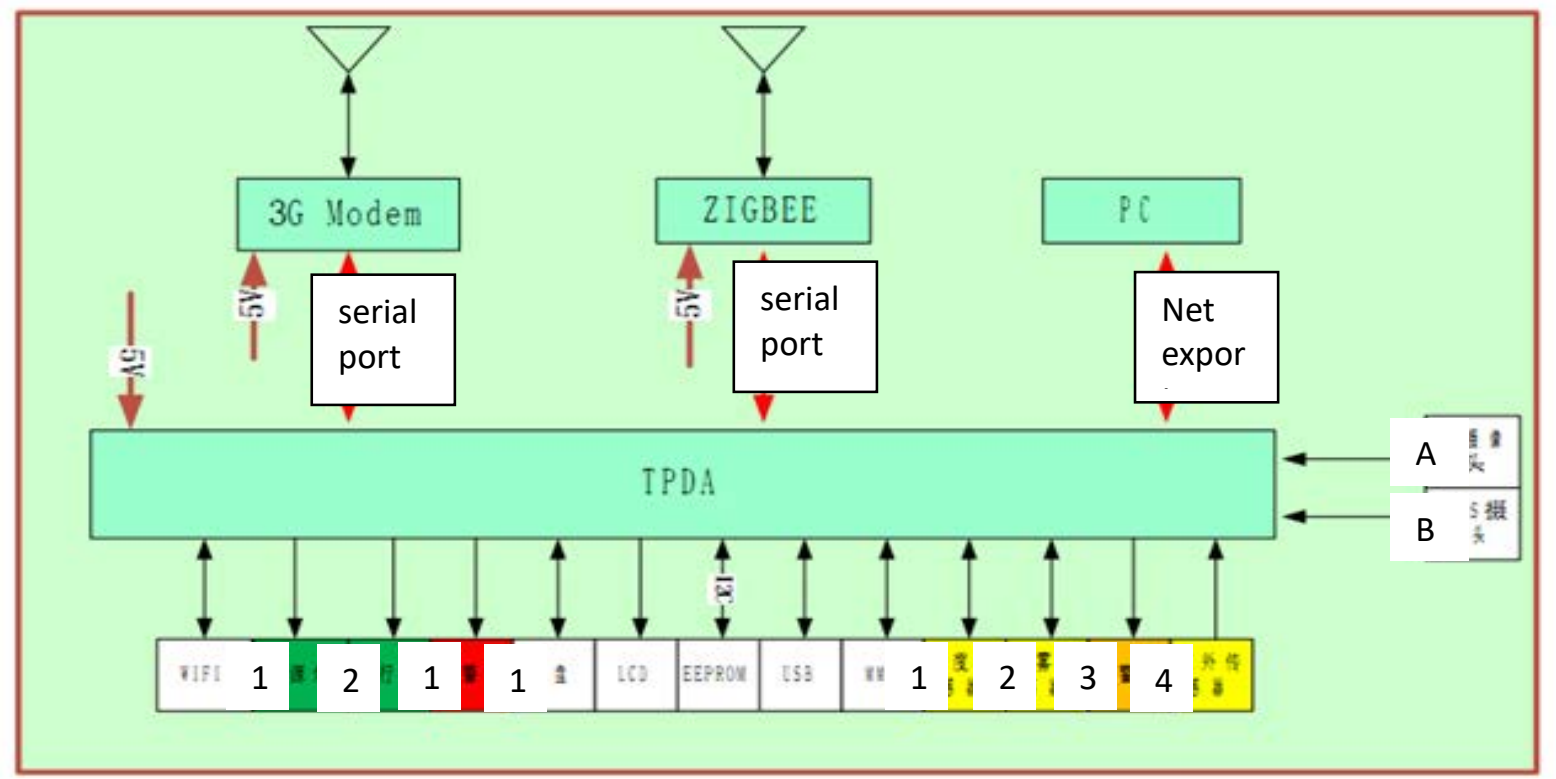

Figure 1: Smart home system hardware framework diagram 
Intelligent plant anti-theft system is generally composed of TPAD, 3GModem, ZIGBEE, PC three units, the hardware framework as shown in figure 1. Each unit (except 3GModem) has the corresponding software running. Each unit power supply board is currently a separate power supply, without the use of PMIC for effective management.

The functions of each hardware interface are described as follows:

(1) operation and maintenance plan

Operation and maintenance of the plane, including system information monitoring, equipment control, software upgrades, such as the configuration and control by the host computer, the hardware interface as shown in figure 2.

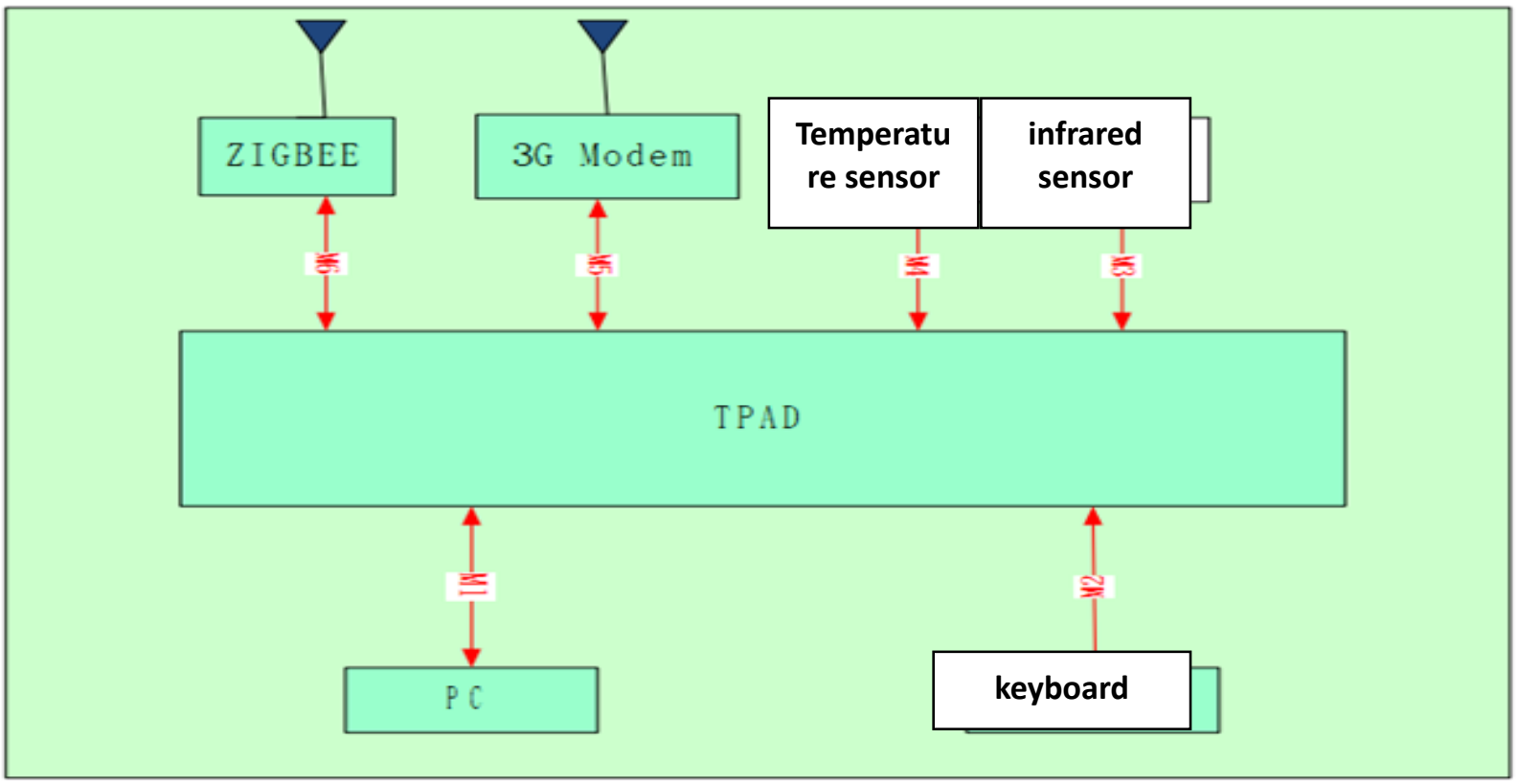

Figure 2: Schematic diagram of the hardware interface of operation and maintenance In figure 2,each maintenance interface is described below:

--M1: operation and maintenance configuration parameters, self report, alarm information, processor status, version query, Ethernet interface;

--M2: operation and maintenance parameters configuration, key keyboard;

--M3: operation and maintenance to obtain the infrared state, signal line;

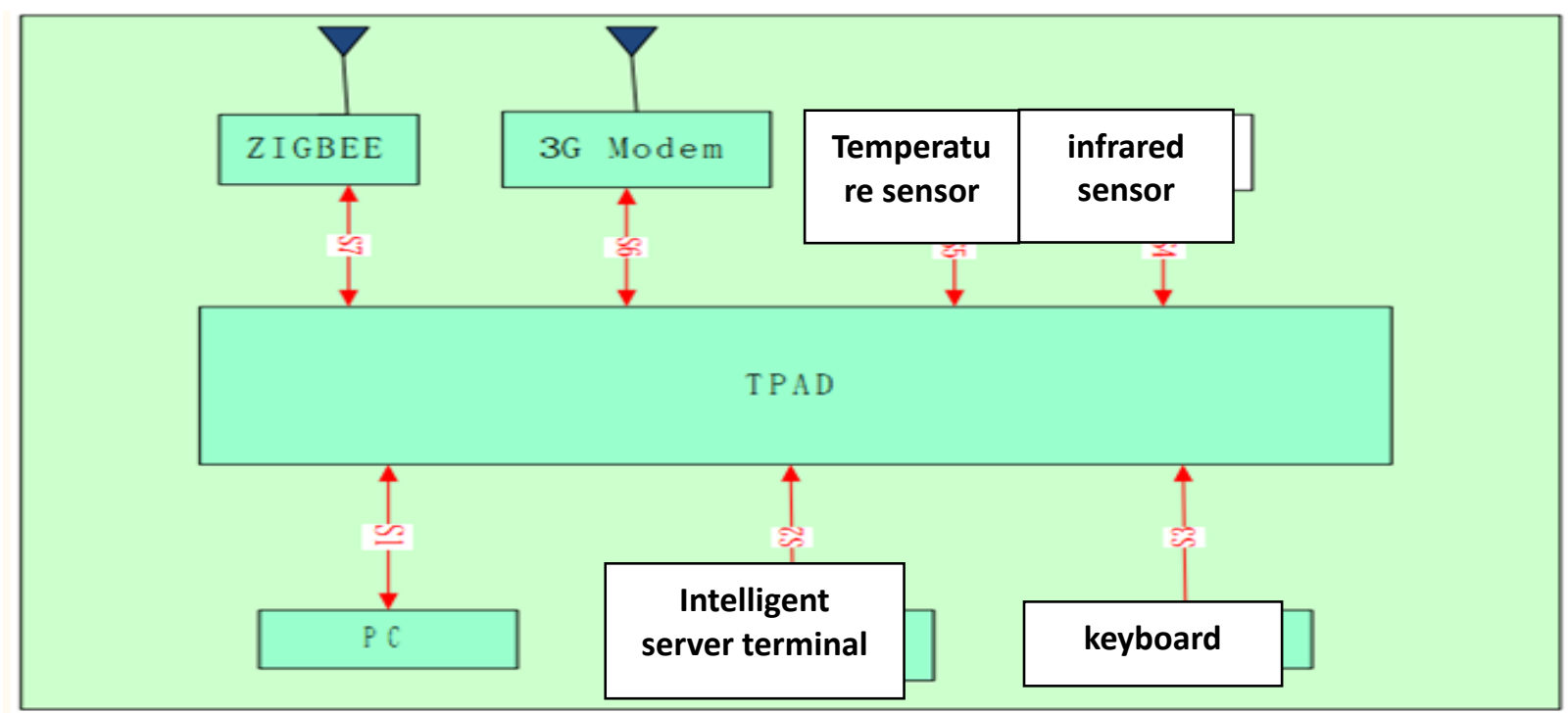

Figuere 3: Hardware interface diagram of control plane

(2) control plane

After the system is powered up, the monitoring software is started, the temperature sensor, 
smoke sensor and infrared sensor are monitored.

At the same time, monitor and deal with the request of the host computer and the intelligent server terminal, and make corresponding processing. The hardware interface is shown in figure 3.

Figure 3 shows the implementation of each control interface as follows:

--S1: the system monitors and processes the request of the host computer;

--S2: system monitoring and processing of intelligent server terminal configuration request and query information, LCD;

--S3: system to accept and process keyboard input information, keyboard keys;

--S4: system blocking or asynchronous notification to monitor the status of infrared sensors, signal lines;

--S5: the system monitors the temperature sensor information regularly;

--S6: system and 3GModem data communication, RS232 interface;

--S7: system and ZIGBEE data communication, RS232 interface.

\section{Software function division}

a. software architecture

4.

Smart home system software includes PC software and embedded software. As shown in figure

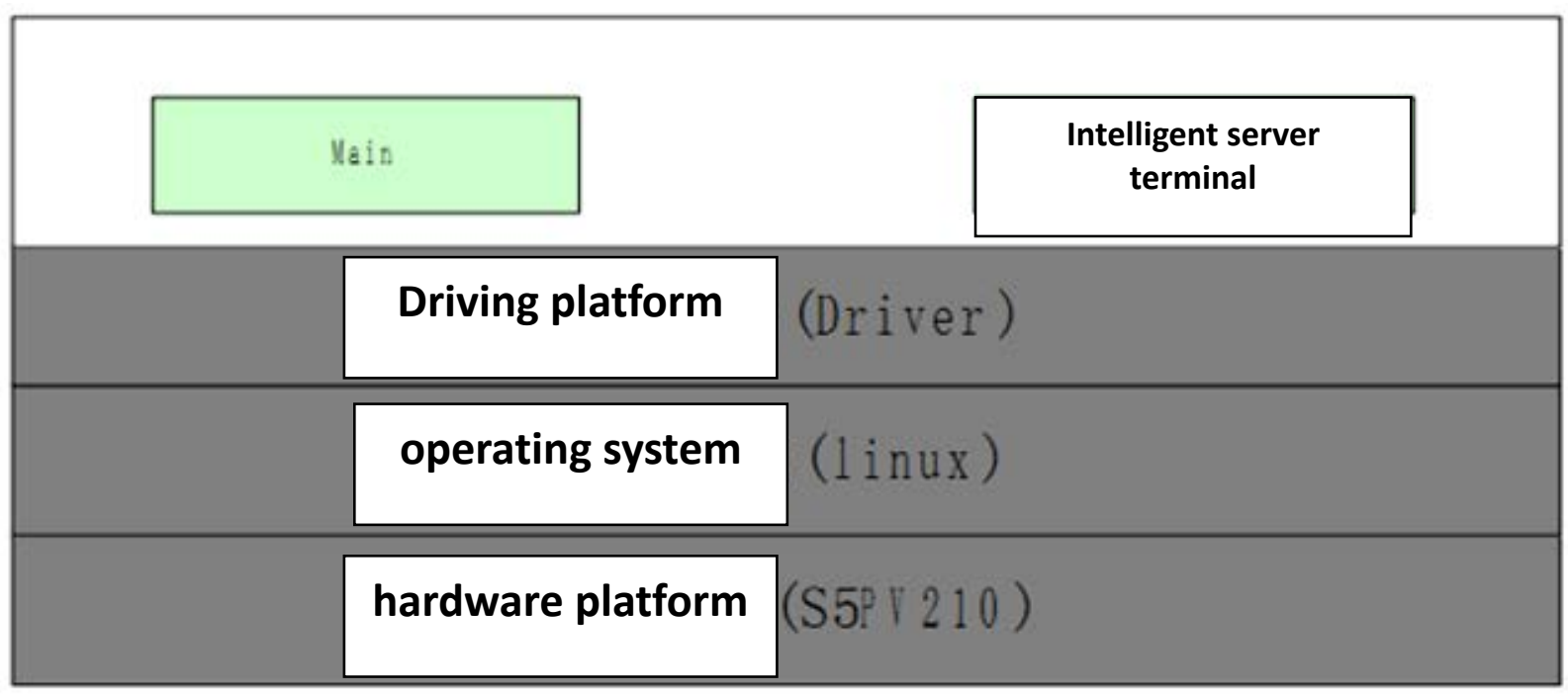

Figure 4.

b. Software subsystem Division

According to the specific functions of different levels of software, the software is divided into modules:

(1) PC application software (smart client)

(2) embedded application software

1)(Main): monitoring the temperature, smoke, infrared sensor, and the corresponding treatment of abnormal function, while listening and processing PC software to send requests

2) intelligent server terminal: display and configure system information;

3) (Main) ZIGBEE: processing of signaling under.

c. Software operating environment and development platform

(1) embedded software

S5PV210 equipped with embedded Linux operating system, debugging software environment Ubuntu, the compiler uses arm-linux-gcc (4.4.6), Eclipse3.7.

(2) smart client

Operating system: Ubuntu

Development environment: QT4

Auxiliary software: qtcreator, qtdesigner

Network protocol: UDP 
Network environment: Ethernet

\section{Conclusion}

At present, intelligent control has been widely applied in many fields of industry, agriculture, military and other practical application problems cannot be solved by traditional control a lot has been solved, showing a strong vitality and development prospects. It will develop with the development of expert system, fuzzy control and neural network. I believe that the future of industrial production must be intelligent control of the world. Intelligent control of the production process will be more potential.

\section{References}

[1] Zhang Xiaomei, Wang Jun Zi, et al. Intelligent control theory and application [M]. Beijing: Tsinghua University press,.2012. (in Chinese)

[2] Huang Xiaoming, Wang Xiaobao, Tan Wen. Overview of intelligent control theory [J]. civil engineering machine, 2014 (3): 30-32.

[3] Song Weng. Introduction of intelligent control technology [M]. Beijing: National Defence Industry Press, 2013

[4] Zhang Si Hui, Tu Jian, neural network control [M]. Beijing: China Machine Press, 2008

[5] Hu Jiangxi. Intelligent control system and application [M]. Beijing: China Electric Power Press, 2011

[6] Zhang Wen, Erwin, Liu et al. Intelligent control and its application [J]. Journal of Chongqing University of Posts and Telecommunications (NATURAL SCIENCE EDITION), 2012 (6): 376-381.

[7] Chen Guo-ming \& William J.Straosta. Intelligent control theory and Application [M]. Shanghai: Shanghai Foreign Language Education Press, 2012.

[8] Kramsch, C.Introduction to intelligent control technology [M]. New York: Doubledny and Company, 2009.

[9] Mitchell, C.Intelligent control system and its application[M].Beijing:China Machine Press, 2010.

[10] Vamer I \& L. Beamer.Introduction to intelligent control technology [M]. Shanghai: China Electric Power Press, 2011.

[11] H Holec, Overview of intelligent control theory (Oxford: Pergamum Press, 2011), 22. 